\title{
An intrinsic anisotropy in the angular distribution of gamma-ray bursts
}

\author{
L.G. Balázs ${ }^{1}$, A. Mészáros ${ }^{1,2,3}$, I. Horváth ${ }^{4}$, and R. Vavrek ${ }^{1}$ \\ 1 Konkoly Observatory, Budapest, Box. 67, H-1505 Hungary \\ e-mail: balazs@ogyalla.konkoly.hu,vavrek@ogyalla.konkoly.hu \\ 2 Department of Astronomy, Charles University, V Holešovičkách 2, CZ-180 00 Prague 8, Czech Republic \\ e-mail: meszaros@mbox.cesnet.cz \\ 3 European Southern Observatory, Karl-Schwarzschild-Strasse 2, Garching bei München, Germany \\ 4 Department of Physics, BJKMF, Budapest POB.12, H-1456 Hungary \\ e-mail: hoi@rmki.kfki.hu
}

Received December 18, 1998; accepted March 10, 1999

\begin{abstract}
The anisotropy of the sky distribution of 2025 gamma-ray bursts (GRBs) collected in Current BATSE catalog is confirmed. It is shown that the quadrupole term being proportional to $\sim \sin 2 b \sin l$ is non-zero with a probability $99.9 \%$. The occurrence of this anisotropy term is then supported by the binomial test even with the probability $99.97 \%$. It is also argued that this anisotropy cannot be caused exclusively by instrumental effects due to the non-uniform sky exposure of BATSE instrument; there should exist also some intrinsic anisotropy in the angular distribution of GRBs. Separating GRBs into short and long subclasses, it is shown that the 251 short ones are distributed anisotropically, but the 681 long ones seem to be distributed still isotropically. The 2-sample KolmogorovSmirnov test shows that they are distributed differently with a $98.7 \%$ probability. The character of anisotropy suggests that the cosmological origin of short GRBs further holds, and there is no evidence for their Galactical origin. The work in essence contains the key ideas and results of a recently published paper (Balázs et al. 1998), to which the new result following from the 2-sample KolmogorovSmirnov test is added, too.
\end{abstract}

Key words: large-scale structure of Universe - gammarays: bursts

\section{Mathematical considerations}

From the mathematical point of view, the necessary condition for the isotropy is the stochastic independency

Send offprint requests to: L.G. Balázs of the sky distribution of the bursts on their observed physical properties. This means that, if $f\left(b, l, x_{1}, \ldots, x_{n}\right)$ $\mathrm{d} F \mathrm{~d} x_{1} \ldots \mathrm{d} x_{2}$ is the probability of finding an object in the $\mathrm{d} F=\cos b \mathrm{~d} l \mathrm{~d} b$ infinitesimal solid angle and in the $\left(x_{1}, x_{1}+\mathrm{d} x_{1}, \ldots, x_{n}, x_{n}+\mathrm{d} x_{n}\right)$ interval, one must have

$f\left(l, b, x_{1}, \ldots, x_{n}\right)=\omega(l, b) g\left(x_{1}, \ldots, x_{n}\right)$.

Here $0 \leq l \leq 360^{\circ},-90^{\circ} \leq b \leq 90^{\circ}$ give the celestial positions in Galactical coordinates, $x_{n}(n \geq 1)$ measure the physical properties (peak fluxes, fluences, durations, etc.) of GRBs and $g$ is their probability density. One may assume the fulfilment of this equation for GRBs.

In the case of spatial isotropy, assuming that the detection probability does not depend on the celestial direction, one has: $\omega(l, b)=1 /(4 \pi)$. In general case one may decompose the function $\omega(b, l)$ into the spherical harmonics. One obtains:

$$
\begin{gathered}
\omega(b, l)=(4 \pi)^{-1 / 2} \omega_{0}- \\
(3 /(4 \pi))^{1 / 2}\left(\omega_{1,-1} \cos b \sin l-\omega_{1,1} \cos b \cos l+\omega_{1,0} \sin b\right)+ \\
(15 /(16 \pi))^{1 / 2}\left(\omega_{2,-2} \cos ^{2} b \sin 2 l+\omega_{2,2} \cos ^{2} b \cos 2 l-\right. \\
\left.\omega_{2,-1} \sin 2 b \sin l-\omega_{2,1} \sin 2 b \cos l\right)+ \\
(5 /(16 \pi))^{1 / 2} \omega_{2,0}\left(3 \sin ^{2} b-1\right)+\text { higher order harm. }
\end{gathered}
$$

The first term on the right-hand side is the monopole term, the following three ones are the dipole terms, the following five ones are the quadrupole terms. Since $\omega$ is constant for isotropic distribution, on the right-hand side any terms, except for $\omega_{0}$, should be identically zeros. To test this hypothesis one has to compute the values of the corresponding spherical harmonic at the celestial positions of the observed GRBs and apply, e.g., the Student test in order to see that the mean of the computed values significantly differs from zero. 
A further trivial consequence of the isotropy is the expected equal number of bursts in celestial regions of equal areas. For example, one may divide the celestial sphere into two equal areas, e.g., taking those regions in which the sign of a given harmonic is either positive or negative, respectively. Then one may compare the number of GRBs in these regions by the standard binomial (Bernoulli) test. The details (together with the relevant references) of this test and also of the test based on the spherical harmonics are discussed in Balázs et al. (1998).

\section{On the existence of the intrinsic anisotropy}

In order to test the isotropy of 2025 GRBs we test the three dipole and five quadrupole terms. One obtains that, except for the terms defined by $\omega_{2,-1}$ and $\omega_{2,-2}$, the remaining six terms may still be taken to be zero. This means that there is a clear anisotropy defined by term $\sim \sin 2 b \sin l$. The probability that this term is zero is $0.1 \%$. In addition, the second quadrupole term being proportional to $\cos ^{2} b \sin 2 l$ is non-zero, too, with the probability $0.6 \%$.

A straightforward counting of GRBs in those regions of equal areas, where $\sin 2 b \sin l$ has either positive or negative signs, respectively, shows that 930 GRBs are in the first area and 1095 are in the second one. Taking $p=0.5$ probability for the binomial (Bernoulli) test, one obtains a $0.03 \%$ probability that this detected distribution is caused by a chance. The observed distribution of all GRBs on sky is anisotropic with a certainty.

Instrumental effects of BATSE instrument should also play a role, since the sky exposure of BATSE instrument is non-uniform. The known dependence of the detection probability of this instrument predicts a similar kind of anisotropy. Hence, the question is the following: is this anisotropy caused either exclusively by the non-uniform sky-exposure function of BATSE instrument, or is there also an intrinsic anisotropy in the distribution of GRBs?

To clarify the situation we divided the GRBs into two groups according to their durations $T_{90}$. We excluded the dimmest GRBs. Then, 932 GRBs were separeted into the "short" ones (251 GRBs; $\left.T_{90}<2 \mathrm{~s}\right)$, and "long" ones (681 GRBs; $T_{90}>2 \mathrm{~s}$ ).

Dividing the sky into the two equal areas, as described above, we obtain a different behaviour for the short and long GRBs, respectively (see Table 1).

Table 1 shows that the short GRBs are further distributed anisotropically; there is a smaller than $1 \%$ probability of isotropy. On the other hand, the long GRBs can still be distributed isotropically. Up to this point all results mentioned in this section, were also written down in Balázs et al. (1998) together with the relevant references.

The application of the 2-sample Kolmogorov-Smirnov test (Press et al. 1992) on $\omega_{2,-1}$ shows that the significance of the difference among the samples of short and
Table 1. Results of the binomial test of subsamples of GRBs with different durations. $N$ is the number of GRBs at the given subsample, $k_{\mathrm{obs}}$ is the observed number GRBs at the first area for this subsample, and \% is the probability in percentages that the assumption of isotropy still holds

\begin{tabular}{ccccc}
\hline sample & $N$ & $k_{\text {obs }}$ & $\left(N-k_{\text {obs }}\right)$ & $\%$ \\
\hline all GRBs & 932 & 430 & 502 & 2.0 \\
$T_{90}<2 \mathrm{~s}$ & 251 & 103 & 148 & 0.55 \\
$T_{90}>2 \mathrm{~s}$ & 681 & 327 & 354 & 32 \\
\hline
\end{tabular}

long GRBs is $98.7 \%$. The short and long ones are obviously distributed differently with a probability $98.7 \%$. Note here that this important quantification of the different behaviour of two subclasses is a new result not presented in Balázs et al. (1998).

We mean that these values confirm the expectation that there must exist some intrinsic anisotropy in the distribution of GRBs. Once there were an exclusive instrumental origin of the anisotropy all GRBs, the character of anisotropy should be the same for both types of GRBs; there should exist no difference among the short and long samples. Of course, the character of anisotropy is quite different than expected for the Galactical origin. For this one would need a clear non-zero $\omega_{2,0}$ spherical harmonic. Hence, there is no doubt concerning the cosmological origin of GRBs.

\section{Conclusions}

1. The distribution of 2025 GRBs is anisotropic with a $99.97 \%$ probability.

2. Separating GRBs into the two classes it is shown that the short ones are distributed anisotropically with a higher than $99 \%$ probability, but the long ones can still be distributed isotropically. There is a $98.7 \%$ probability that the short and long GRBs are distributed differently.

3. This different behaviour of two samples cannot be caused exclusively by instrumental effects; there must exist also some intrinsic anisotropy, too.

4. The cosmological origin is not queried by this anisotropy.

Acknowledgements. A.M. thanks for the kind hospitality at ESO. R.V. acknowledges the valuable discussions with I. Domsa. This article was partly supported by OTKA grants T024027 (L.G.B.), F29461 (I.H.), by GAUK grant 36/97 and by GAČR grant 202/98/0522 (A.M.).

\section{References}

Balázs L.G., Mészáros A., Horváth I., 1998, A\&A 339, 1 Press W.H., Teukolsky S.A., Vetterling W.T., Flannery B.P., Numerical Recipes in Fortran, Second Edition. Cambridge University Press, Cambridge, 1992, Chapt. 14.3 